\title{
UNIVERSITY OF LUND RADIOCARBON DATES XI
}

\author{
SÖREN HÅKANSSON
}

Racliocarbon Dating Laboratory, Department of Quaternary Geology,

University of Lund, Sweden

INTRODUCTION

Most of the ${ }^{1+} \mathrm{C}$ measurements reported here were made between October 1976 and October 1977. Equipment, measurement, and treatment of samples are as reported previously (R, 1968, v 10, p 36-37; 1976, v 18, p 290).

Age calculations are based on a contemporary value equal to 0.950 of the activity of NBS oxalic acid standard and on the conventional half-life for ${ }^{14} \mathrm{C}$ of $5568 \mathrm{yr}$. Results are reported in years before 1950 (years $\mathrm{BP})$. Errors quoted $( \pm 1 \sigma)$ include standard deviations of count rates for the unknown sample, contemporary standard, and background. When measured activity is less than $2 \sigma$ above background, minimum age is given. Basis for calculation of age limit is measured net activity plus $3 \sigma$. If net activity is negative, only $+3 \sigma$ is used for age limit.

Corrections for deviations from $\delta^{13} \mathrm{C}=-25.0^{\%} \%$ in the PDB scale are applied for all samples; also for marine shells. The apparent age for marine material must be subtracted from our dates on such samples.

The remark, "undersized; diluted", in Comments means the sample did not produce enough $\mathrm{CO}_{2}$, to fill the counter to normal pressure and "clead" $\mathrm{CO}_{2}$ from anthracite was introduced to make up the pressure. "\% sample" indicates amount of $\mathrm{CO}_{2}$ derived from the sample present in the diluted counting gas; the rest is "dead" $\mathrm{CO}_{2}$. Organic carbon content reported for bone samples is calculated from yield of $\mathrm{CO}_{2}$ by combustion of gelatine remaining after treatment. Organic carbon lost during treatment is not included in calculated percentage.

The description of each sample is based on information provided by the submitter.

ACKNOWLEDGMENTS

The author thanks Kerstin Lundahl for sample preparation and routine operation of the dating equipment, and $\mathrm{R}$ Ryhage and his staff at the mass-spectrometric laboratory of Karolinska Inst, Stockholm, for the ${ }^{13} \mathrm{C}$ analyses.

\section{SAMPLE DESCRIPTIONS}

I. GEOLOGIC SAMPLES

\section{Håkulls mosse series}

\section{A. Sweden}

Sediment from bog Håkulls mosse on hill ridge Kullaberg, NW Scania $\left(56^{\circ} 17^{\prime} \mathrm{N}, 12^{\circ} 31^{\prime} \mathrm{E}\right)$. Alt ca $125 \mathrm{~m}$. Coll 1975 and subm by B E Berglund, Dept Quaternary Geol, Univ Lund. Samples are from core taken with Livingstone sampler, $100 \mathrm{~mm}$ diam. Depths refer to bog sur- 
face. Site and older pollen diagram described earlier (Berglund, 1971). No carbonate content. All samples pretreated with $\mathrm{HCl}$; Nos. 5, 6, and 8 also with $\mathrm{NaOH}$.

Lu-1262. Håkulls mosse 1, 866 to $868 \mathrm{~cm}$

Clay gyttja. Beginning of Bölling zone.

Lu-1263. Håkulls mosse 2, 859 to $861 \mathrm{~cm}$

Clay gyttja. Middle of Bölling zone.

Lu-1264. Håkulls mosse 3, 854 to $856 \mathrm{~cm}$

Clay gyttja. Later part of Bölling zone.

Lu-1265. Håkulls mosse 4, 847 to $849 \mathrm{~cm}$

Clay gyttja. Older Dryas zone.

Lu-1331. Håkulls mosse 5, 806 to $808 \mathrm{~cm}$

Gyttja. Transition Alleröd/Younger Dryas.

Lu-1332. Håkulls mosse 6, 804 to $806 \mathrm{~cm}$

Clay gyttja. Transition Alleröd/Younger Dryas.

Lu-1333. Håkulls mosse 7,753 to $755 \mathrm{~cm}$

Clay gyttja. Later part of Younger Dryas zone.

Lu-1334. Håkulls mosse 8, 730 to $732 \mathrm{~cm}$

Clay gyttja. Transition Younger Dryas/Pre-Boreal.

Lu-1335. Håkulls mosse 9, 724 to 726cm

Clay gyttja. Transition Younger Dryas/Pre-Boreal.

Lu-1336. Håkulls mosse 10,653 to $655 \mathrm{~cm}$

Gyttja. Later part of Pre-Boreal zone.
$13,020 \pm 135$ $\delta^{13} C=-23.7 \%$

$12,660 \pm 125$ $\delta^{13} \mathrm{C}=-24.9 \%$

$12,440 \pm 120$ $\delta^{13} \mathrm{C}=-22.9 \%$

$12,150 \pm 120$ $\delta^{13} \mathrm{C}=-22.4 \%$

$11,240 \pm 110$ $\delta^{13} \mathrm{C}=-24.4 \%$

$11,050 \pm 110$ $\delta^{13} C=-24.6 \%$

$10,430 \pm 105$ $\delta^{13} C=-24.3 \%$ o

$10,240 \pm 100$ $\delta^{13} C=-26.0 \%$

$10,110 \pm 100$ $\delta^{13} C=-26.9 \%$

$$
9310 \pm 95
$$

$\delta^{13} \mathrm{C}=-29.6 \%$

\section{Jämjö series}

Wood samples from silty and sandy deposits in valley of rivulet Åbyån, N of Jämjö, E Blekinge. Coll 1976 and subm by S Björck, Dept Quaternary Geol, Univ Lund. Dating is part of study of influence of sea-level changes on erosion and accumulation in valley and of relation between solifluction material and climatic and vegetational changes and/ or human influence in area. Samples pretreated with $\mathrm{HCl}$ and $\mathrm{NaOH}$. 
Lu-1261. Jämjö church

$$
\begin{array}{r}
1570 \pm \mathbf{5 0} \\
\delta^{1.3} C=-26.8 \% \text { \% }
\end{array}
$$

Wood fragments (Almus sp) id by $\mathbf{T}$ Bartholin from humic sand and silt $1.7 \mathrm{~m}$ below surface at Jämjö church $\left(56^{\circ} 11^{\prime} 30^{\prime \prime} \mathrm{N}, 15^{\circ} 50^{\prime} 5^{\prime \prime} \mathrm{E}\right)$. Stratified sand and silt from $2.5 \mathrm{~m}$ below surface and further down.

\section{Lu-1296. Hagalund 6}

$$
\begin{array}{r}
4890 \pm 70 \\
\delta^{13} C=-26.5 \% \text { o }
\end{array}
$$

Unid wood fragments from sand $2.25 \mathrm{~m}$ below surface at Hagalund 6 , ca $1 \mathrm{~km} \mathrm{~N}$ of Jämjö church $\left(56^{\circ} 12^{\prime} \mathrm{N}, 15^{\circ} 50^{\prime} 15^{\prime \prime} \mathrm{E}\right)$. Silt with clay layers from $3.5 \mathrm{~m}$ below surface and further down. Comment: sample undersized; diluted; $91 \%$ sample.

\section{Sämbosjön series}

Sediment from Lake Sämbosjön, Halland, SW Sweden $\left(57^{\circ} 10^{\prime} \mathrm{N}\right.$, $12^{\circ} 25^{\prime} \mathrm{E}$ ). Alt $38 \mathrm{~m}$; area $0.2 \mathrm{sq} \mathrm{km}$; max depth $6 \mathrm{~m}$. Coll 1976 and subm by G Digerfeldt, Dept Quaternary Geol, Univ Lund. Dating is part of study of Flandrian development of lake and vegetational history of surrounding region. Samples come from profile in central part of lake (Livingstone sampler, diam $100 \mathrm{~mm}$ ). Water depth $6 \mathrm{~m}$ at sampling point. Depths given are below sediment surface. Lu-1267 through Lu-1271 pretreated with $\mathrm{HCl}$ only. All other samples pretreated with $\mathrm{HCl}$ and $\mathrm{NaOH}$.

\section{Lu-1270. Sämbosjön, 610 to $615 \mathrm{~cm}$}

$$
\begin{array}{r}
9860 \pm 95 \\
\delta^{13} C=-22.9 \%
\end{array}
$$

Clay gyttja.

\section{Lu-1267. Sämbosjön, 595 to $600 \mathrm{~cm}$}

$$
\begin{array}{r}
9390 \pm 95 \\
\delta^{13} C=-27.2 \% \text {. }
\end{array}
$$

Slightly clayey detritus gyttja.

Lu-1268. Sämbosjön, 555 to $560 \mathrm{~cm}$

$$
\begin{array}{r}
9170 \pm 90 \\
\delta^{1.3} C=-27.2 \%
\end{array}
$$

Slightly clayey detritus gyttja.

\section{Lu-1269. Sämbosjön, 515 to $520 \mathrm{~cm}$}

$$
\begin{array}{r}
9190 \pm 90 \\
\delta^{13} C=-26.4 \% \text { o }
\end{array}
$$

Slightly clayey detritus gyttja.

Lu-1271. Sämbosjön, 460 to $465 \mathrm{~cm}$

$$
\begin{array}{r}
\mathbf{8 2 8 0} \pm \mathbf{8 5} \\
\delta^{13} C=-25.6 \%
\end{array}
$$

Detritus gyttja.

\section{Lu-1272. Sämbosjön, 420 to $425 \mathrm{~cm}$}

$$
\begin{array}{r}
\mathbf{7 8 2 0} \pm \mathbf{8 5} \\
\delta^{1.3} \mathrm{C}=-28.3 \%
\end{array}
$$

Detritus gyttja. Comment: part of this sample was dated without $\mathrm{NaOH}$ treatment at $7710 \pm 80$. 
Lu-1273. Sämbosjön, 380 to $385 \mathrm{~cm}$

Detritus gyttja.

Lu-1274. Sämbosjön, 340 to $345 \mathrm{~cm}$

Detritus gyttja.

Lu-1275. Sämbosjön, 300 to $305 \mathrm{~cm}$

Detritus gyttja.

Lu-1276. Sämbosjön, 260 to $265 \mathrm{~cm}$

Detritus gyttja.

Lu-1277. Sämbosjön, 220 to $225 \mathrm{~cm}$

Detritus gyttja.

Lu-1278. Sämbosjön, 180 to $185 \mathrm{~cm}$

Detritus gyttja.

Lu-1279. Sämbosjön, 140 to $145 \mathrm{~cm}$

Detritus gyttja.
$6740 \pm 75$

$\delta^{13} \mathrm{C}=-28.5 \%$

$$
6220 \pm 70
$$$$
\delta^{1.3} \mathrm{C}=-28.3 \%
$$

$5200 \pm 65$
$\delta^{13} C=-27.6 \%$

$4270 \pm 60$
$\delta^{13} C=-28.4 \%$

$3130 \pm 55$

$\delta^{1.3} \mathrm{C}=-28.5 \%$

$\mathbf{2 4 4 0} \pm \mathbf{5 5}$

$\delta^{13} C=-28.3 \%$

$\mathbf{2 0 3 0} \pm \mathbf{5 0}$

$\delta^{1.3} \mathrm{C}=-28.4 \%$

\section{Hullsjön series}

Sediment from Lake Hullsjön, Västergötland, central Sweden $\left(58^{\circ}\right.$ $\left.17^{\prime} \mathrm{N}, 12^{\circ} 23^{\prime} \mathrm{E}\right)$. Alt $38 \mathrm{~m}$; area $1.8 \mathrm{sq} \mathrm{km}$; max depth $1.5 \mathrm{~m}$. Coll 1976 and subm by $G$ Digerfeldt. Dating is part of study of Late Flandrian development of lake and vegetational history of surrounding region. Samples come from profile in central part of lake (Livingstone sampler, diam $60 \mathrm{~mm}$ ). Water depth $1.5 \mathrm{~m}$ at sampling point. Depths given are below sediment surface. All samples pretreated with $\mathrm{HCl}$.

\section{Lu-1305. Hullsjön, 380 to $385 \mathrm{~cm}$}

Clay gyttja.

Lu-1306. Hullsjön, 355 to $360 \mathrm{~cm}$

Clayey detritus gyttja.

Lu-1307. Hullsjön, 295 to $300 \mathrm{~cm}$

Clayey detritus gyttja.

Lu-1308. Hullsjön, 255 to $260 \mathrm{~cm}$

Clay gyttja.

$$
\begin{array}{r}
\mathbf{5 4 4 0} \pm \mathbf{7 0} \\
\delta^{13} C=-27.8 \% / 6
\end{array}
$$

$$
\begin{array}{r}
4950 \pm 65 \\
\delta^{1 .} C=-28.0 \%
\end{array}
$$

$$
\begin{array}{r}
\mathbf{3 4 9 0} \pm \mathbf{6 0} \\
\delta^{13} C=-27.5 \%
\end{array}
$$

$$
\begin{array}{r}
2670 \pm \mathbf{5 5} \\
\delta^{1.3} C=-27.4 \%
\end{array}
$$


Lu-1309. Hullsjön, 195 to $200 \mathrm{~cm}$

$$
\begin{array}{r}
\mathbf{1 4 1 0} \pm \mathbf{5 0} \\
\delta^{1.5} C=-28.1 \% \\
\mathbf{7 6 0} \pm \mathbf{5 0} \\
\delta^{1.5} \mathrm{C}=-28.1 \% \mathrm{C}
\end{array}
$$

Clayey detritus gyttja.

Clay gyttja.

\section{Långsjön series}

Sediment from Lake Långsjön, Stockholm $\left(59^{\circ} 16^{\prime} \mathrm{N}, 17^{\circ} 58^{\prime} \mathrm{E}\right)$. Alt $31 \mathrm{~m}$; area $0.3 \mathrm{sq} \mathrm{km}$; $\max$ depth $3.1 \mathrm{~m}$. Coll 1976 and subm by $\mathrm{G}$ Digerfeldt. Dating is part of study of Late Flandrian development of lake and vegetational history of surrounding region. Samples come from profile in deepest part of lake (Livingstone sampler, diam $60 \mathrm{~mm}$ ). Water depth $3.1 \mathrm{~m}$ at sampling point. Depths given are below sediment surface. All samples pretreated with $\mathrm{HCl}$.

\section{Lu-1368. Långsjön, 481 to $486 \mathrm{~cm}$}

$$
\begin{array}{r}
\mathbf{4 8 8 0} \pm \mathbf{6 5} \\
\delta^{13} C=-25.4 \% o
\end{array}
$$

Slightly clayey algae gyttja. Just above isolation level.

\section{Lu-1338. Långsjön, 471 to $476 \mathrm{~cm}$}

Slightly clayey algae gyttja.

Lu-1339. Långsjön, 421 to $426 \mathrm{~cm}$

Slightly clayey detritus gyttja.

Lu-1340. Långsjön, 371 to $376 \mathrm{~cm}$

Detritus gyttja.

Lu-1341. Långsjön, 321 to $326 \mathrm{~cm}$

Detritus gyttja.

Lu-1342. Långsjön, 271 to $276 \mathrm{~cm}$

Detritus gyttja.

Lu-1343. Långsjön, 221 to $226 \mathrm{~cm}$

Detritus gyttja.

Lu-1344. Långsjön, 171 to $176 \mathrm{~cm}$

Detritus gyttja.

Lu-1345. Långsjön, 121 to $126 \mathrm{~cm}$

Detritus gyttja.

$$
\begin{array}{r}
\mathbf{4 9 4 0} \pm \mathbf{6 5} \\
\delta^{13} C=-24.8 \% \circ \\
\mathbf{4 4 5 0} \pm \mathbf{6 5} \\
\delta^{13} C=-27.2 \%
\end{array}
$$

$$
4180 \pm 60
$$$$
\delta^{13} \mathrm{C}=-27.7 \%
$$

$4070 \pm 60$
$\delta^{13} C=-28.1 \%$ o

$\mathbf{3 7 8 0} \pm \mathbf{6 0}$
$\delta^{13} \mathrm{C}=-28.9 \%$

$$
3370 \pm 60
$$$$
\delta^{1.3} \mathrm{C}=-29.5 \%
$$

$$
\mathbf{2 4 4 0} \pm \mathbf{5 0}
$$$$
\delta^{13} C=-29.6 \%
$$

$$
\mathbf{2 0 4 0} \pm \mathbf{5 0}
$$$$
\delta^{13} \mathrm{C}=-29.9 \%
$$ 
Lu-1366. Långsjön, 95 to $97 \mathrm{~cm}$

Detritus gyttja.

Lu-1367. Långsjön, 70 to $72 \mathrm{~cm}$

Detritus gyttja.

Lu-1297. Innaren
$1720 \pm 50$ $\delta^{1.3} \mathrm{C}=-29.9 \%$

$$
990 \pm 50
$$$$
\delta^{1.3} C=-29.7 \%
$$

$\begin{aligned} & >\mathbf{4 0 , 6 0 0} \\ \delta^{13} \mathrm{C}= & -26.9^{\prime} / \mathrm{CO}\end{aligned}$

Peat from ca $5 \mathrm{~m}$ below surface found by well-digging near $\mathrm{S}$ end of Lake Innaren, South Swedish Upland ( $56^{\circ} 58^{\prime} 10^{\prime \prime} \mathrm{N}, 14^{\circ} 58^{\prime} 55^{\prime \prime} \mathrm{E}$ ). Alt ca $180 \mathrm{~m}$. Coll Oct 1976 by U Lettevall; subm by G Digerfeldt.

\section{Fjällsjön Series II}

Sediment from Lake Fjällsjön, 3km SE of Sandhult church, central Västergötland (57 $\left.45^{\prime} 6^{\prime \prime} \mathrm{N}, 12^{\circ} 51^{\prime} 40^{\prime \prime} \mathrm{E}\right)$. Coll 1975 and subm by A Hilldén, Dept Quaternary Geol, Univ Lund. Dated as complement to Fjällsjön series (R, 1977, v 19, p 427-428). Depths refer to water surface. Water depth at sampling point ca $4 \mathrm{~m}$. Pretreated with HCl.

Lu-1363. Fjällsjön 1, 839 to $842 \mathrm{~cm} \quad 11,700 \pm 125$ $\delta^{13} \mathrm{C}=-21.9 \%$

Clay gyttja. Comment: undersized; diluted; $90 \%$ sample.

$\begin{array}{rr}\text { Lu-1364. Fjällsjön 1, } 831 \text { to } 833 \mathrm{~cm} & 11,250 \pm 120 \\ \delta^{13} C=-22.4 \%\end{array}$

Clay gyttja. Comment: undersized; diluted; $91 \%$ sample.

\section{Lu-1392. Ljungsjön, 833 to $837 \mathrm{~cm}$}

$11,160 \pm 125$
$\delta^{13} C=-25.8 \%$

Clay gyttja from Lake Ljungsjön, $8 \mathrm{~km}$ SW of Ulricehamn, central Västergötland $\left(57^{\circ} 44^{\prime} 3^{\prime \prime} \mathrm{N}, 13^{\circ} 18^{\prime} 38^{\prime \prime} \mathrm{E}\right)$. Coll 1975 and subm by A Hilldén. Dated as complement to Ljungsjön series (R, 1977, v 19, p 427). Depth relers to water surface. Pretreated with HCl. Comment: undersized; diluted; $66 \%$ sample. (3 1-day counts.)

\section{Björksjödamm series}

Sediment from mire pool Björksjödamm, $1 \mathrm{~km} \mathrm{~N}$ of Rya, $30 \mathrm{~km} \mathrm{E} \mathrm{of}$

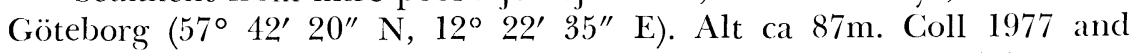
subm by A Hilldén. Samples are from core taken with Livingstone sampler, $100 \mathrm{~mm}$ diam. Depths refer to water surface. Water depth ca $2 \mathrm{~m}$ at sampling point. No carbonate content. Pretreated with HCl. All samples slightly undersized; diluted. Amount of $\mathrm{CO}_{2}$ from sample is given in Comment below as "\% sample".

\section{Lu-1394. Björksjödamm $1+2,842$ to $848 \mathrm{~cm} \quad 12,550 \pm 145$}

Muddy clay and silt. Bölling zone. Comment: $82 \%$ sample. 
Lu-1395. Björksjödamm 3, 836 to $840 \mathrm{~cm}$

Muddy clay. Older Dryas zone. Comment: $88 \%$ sample.

Lu-1396. Björksjödamm 4, 830 to $834 \mathrm{~cm} \quad 12,030 \pm 125$

Muddy clay. Beginning of Alleröd zone. Comment: $97 \%$ sample.

\section{Lu-1393. Björksjödamm 6, 818 to $822 \mathrm{~cm} \quad 11,170 \pm 90$}

$\delta^{13} \mathrm{C}=-2.1 \%$

Muddy clay. Transition Alleröd/Younger Dryas zone. Comment: $88 \%$ sample. (3 1-day counts.)

\section{Lu-1397. Björksjödamm 5, 808 to $812 \mathrm{~cm} \quad 10,830 \pm 120$}

$\delta^{13} C=-24.9 \%$ sample.

Mucldy clay. Beginning of Younger Dryas zone. Comment: $85 \%$

\section{Lu-1362. Ven}

$$
\begin{array}{r}
>\mathbf{4 1 , 6 0 0} \\
\delta^{1.3} C=+0.8 \%
\end{array}
$$

Redeposited marine shell fragments (Mya truncata, Macoma calcarea, and Hiatella arctica) id by K Strand Petersen, Geol Survey Denmark, Copenhagen, from gravelly sand at $+2.5 \mathrm{~m}$ near $\mathrm{S}$ end of Ven $\mathrm{I}$.

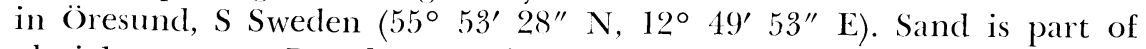
glacial sequence. Dated to obtain maximum age of overlying tills. Coll 1976 and subm by L Adrielsson, Dept Quaternary Geol, Univ Lund. Comment: outer $23 \%$ of shells removed by acid leaching. (3 1-day counts.)

\section{B. Norway}

\section{Varanger Peninsula Series II}

Sediment from lakes on Varanger Peninsula, N Norway. Dated as complement to Varanger Peninsula series (R, 1974, v 16, p 317-318). For other dates from area, see also Angsnes series (R, 1977, v 19, p 431 -432). Coll 1976 and subm by $\mathbf{B}$ Malmström and O Palmér, Dept Phys Geog, Univ Lund. Samples represent transition from minerogenic to organogenic deposits. Depths are below sediment-water interface. Pretreated with HCl. All samples undersized; diluted. Amount of $\mathrm{CO}_{2}$ from sample is given in Comments below as "\% sample".

\section{Lu-1254. Bergelyvand 1, 272 to $280 \mathrm{~cm} \quad 10,420 \pm 160$} $\delta^{1.3} \mathrm{C}=-19.2 \%$

Light greygreen muddy clay. Comment: $55 \%$, sample.

\section{Lu-1258. Bergebyvand 5, 275 to $280 \mathrm{~cm} \quad 10,590 \pm 145$} $\delta^{13} \mathrm{C}=-19.6^{\mathrm{c}} / \mathrm{co}$ counts.)

Light greengray muddy clay. Comment: $51 \%$ sample. (3 1-day 
Lu-1255. Holmf jeldvand, 265 to $270 \mathrm{~cm}$

$$
\begin{array}{r}
9200 \pm \mathbf{1 0 0} \\
\delta^{1.3} \mathrm{C}=-20.8 \% \%
\end{array}
$$

Graygreen clay gyttja. Comment: $86 \%$ sample.

Lu-1256. Ostrevand 3, 200 to $205 \mathrm{~cm} \quad 11,640 \pm 130$ $\delta^{13} \mathrm{C}=-24.1 \%$

Light graygreen muddy clay. Comment: 69\% sample. (3 1-day counts.)

Lu-1257. Ostrevand 4- $1 \mathrm{lb}, 149$ to $157 \mathrm{~cm} \quad 11,550 \pm 140$

$\delta^{13} C=-25.2 \%$ counts.)

Light graygreen muddy clay. Comment: $62 \%$ sample. (3 1 -day

Lu-1259. Stjernevand, 197 to $209 \mathrm{~cm}$

$9370 \pm 150$

Clayey gyttja. Comment: $54 \%$ sample.

\section{Sotra series (I)}

Sediment from small lakes on Sotra I., Hordaland, W Norway. Coll 1976 and subm by K Krzywinski, Hist Mus, Univ Bergen. Dated as part of study of sea-level changes in area. All samples pretreated with $\mathrm{HCl}$ and then separated in acid-precipitated part of $\mathrm{NaOH}$-soluble fraction and insoluble residue (called soluble and residue, respectively, below).

Lu-1353A. Sotra no. 5815, soluble

$$
\begin{array}{r}
9290 \pm \mathbf{9 5} \\
\delta^{13} C=-26.7 \% \text {. }
\end{array}
$$

Lacustrine dy from Lommatjonn $\left(60^{\circ} 15^{\prime} \mathrm{N}, 5^{\circ} 02^{\prime} \mathrm{E}\right)$ from isolation contact formed during Pre-Boreal regression. Threshold alt $+11.4 \mathrm{~m}$.

Lu-1353. Sotra no 5815, residue

Lu-1354A. Sotra no. 5830, soluble

$$
\begin{array}{r}
\mathbf{9 3 4 0} \pm \mathbf{9 5} \\
\delta^{13} C=-27.9 \% \\
\mathbf{7 4 0 0} \pm \mathbf{1 0 0} \\
\delta^{13} C=-28.3 \%
\end{array}
$$

Lacustrine dy from Lommatj $\phi \mathrm{nn}$ from transition to brackish gyttja deposited during Tapes-transgression. Comment: sample undersized; diluted; $68 \%$ sample.

Lu-1354. Sotra no. 5830, residue

$$
\begin{array}{r}
7380 \pm \mathbf{8 0} \\
\delta^{13} C=-29.6 \% \\
\mathbf{5 4 9 0} \pm \mathbf{6 5} \\
\delta^{13} C=-30.0 \%
\end{array}
$$

Lu-1355A. Sotra no. 5884-5, soluble

Lacustrine gyttja from Lommatjonn from isolation contact formed during regression following Tapes-transgression.

Lu-1355. Sotra no. 5884-5, residue

$$
\begin{array}{r}
\mathbf{5 4 0 0} \pm \mathbf{6 5} \\
\delta^{13} \mathrm{C}=-31.0 \%
\end{array}
$$

Comment: sample undersized; diluted; $75 \%$ sample. (3 1-day counts.) 
Lu-1358A. Sotra no. 5940-1, soluble

$$
\begin{array}{r}
4260 \pm 60 \\
\delta^{13} C=-26.6 \%
\end{array}
$$

Lacustrine dy from Midtjonn $\left(60^{\circ} 14^{\prime} \mathrm{N}, 5^{\circ} 02^{\prime} \mathrm{E}\right)$ from isolation contact formed during regression following Tapes-transgression. Threshold alt $+7.9 \mathrm{~m}$.

Lu-1357A. Sotra no. 6109, soluble

$$
\begin{array}{r}
9150 \pm 100 \\
\delta^{1.3} C=-24.5 \% \text { o }
\end{array}
$$

Lacustrine dy from Klokkarvatnet $\left(60^{\circ} 15^{\prime} \mathrm{N}, 5^{\circ} 02^{\prime} \mathrm{E}\right)$ from isolation contact formed during regression before Tapes-transgression. Threshold alt $+6.9 \mathrm{~m}$. Comment: sample undersized; diluted; $89 \%$ sample.

Lu-1356A. Sotra no. 6199, soluble

$$
\begin{array}{r}
3990 \pm \mathbf{6 0} \\
\delta^{13} C=-29.0 \%
\end{array}
$$

Lacustrine dy from Klokkarvatnet from isolation contact formed after Tapes-transgression.

\section{Lu-1359A. Sotra no. 6281, soluble}

$$
4340 \pm 60
$$

$\delta^{13} C=-27.3 \%$

Lacustrine dy from Kaldavatn $\left(60^{\circ} 16^{\prime} \mathrm{N}, 4^{\circ} 58^{\prime} \mathrm{E}\right)$ from isolation contact formed during regression following Tapes-transgression. Threshold alt $+7.55 \mathrm{~m}$.

Lu-1360A. Sotra no. 6358, soluble

$$
\begin{array}{r}
\mathbf{8 0 6 0} \pm \mathbf{8 5} \\
\delta^{13} \mathrm{C}=-25.8 \% \text { 。 }
\end{array}
$$

Lacustrine dy from Kaldavatn from ingression contact formed during Tapes-transgression.

\section{Lu-1361A. Sotra no. 6368, soluble}

$$
\begin{array}{r}
\mathbf{9 3 4 0} \pm \mathbf{9 0} \\
\delta^{13} \mathrm{C}=-24.7 \%
\end{array}
$$

Lacustrine dy from Kaldavatn from isolation contact formed during Pre-Boreal regression.

\section{East Greenland series (VI)}

$$
\text { C. Greenland }
$$

Bivalve shells from emerged marine sediments, and lake sediment samples from Hochstetter Forland, Shannon Ö, and Ardencaple Fjord, NE Greenland. Coll by the Swedish-Danish Expedition of 1976 (C Hjort, L Adrielsson, H Bruch, and J Mikaelsson) and subm as part of study of glaciation chronology, shore-line displacement, and vegetation history. For other East Greenland dates from this lab, see R, 1972, v 14, p $388-390 ; 1973$, v 15, p 504-507; 1974, v 16, p 319-322; 1975, v 17, p 184-187; 1976, v 18, p 301-303. According to Hjort (1973) a sea correction of $-550 \mathrm{yr}$ should be applied to shell dates, below.

\section{Lu-1288. Åsen}

$$
\begin{array}{r}
\mathbf{3 9 , 0 0 0} \begin{array}{l}
+\mathbf{2 4 0 0} \\
-\mathbf{1 8 0 0}
\end{array} \\
\delta^{1.3} C=+1.1 \% \circ
\end{array}
$$

Shell fragments (Mya truncata, Hiatella arctica) from beach gravel overlying laminated silt and sand deposited in front of and dating 
terminal moraine on S Hochstetter Forland ( $\left.75^{\circ} 13^{\prime} \mathrm{N}, 19^{\circ} 55^{\prime} \mathrm{W}\right)$. Alt $90 \mathrm{~m}$. Comment: outer $40 \%$ of shells removed by acid leaching. (3 1-day counts.)

\section{Lu-1289. Mönstedhus}

$$
\begin{array}{r}
9190 \pm 90 \\
\delta^{13} C=-4.7 \%
\end{array}
$$

Shells (Portlandia arctica) from silt at $22 \mathrm{~m}$, underlying delta built up to $53 \mathrm{~m}$ near Mönstedhus, N Hochstetter Forland $\left(75^{\circ} 43^{\prime} \mathrm{N}, 19^{\circ}\right.$ $40^{\prime} \mathrm{W}$ ). Dates deglaciation of area. Comment: no surface leaching; small sample. Shells well preserved with periostracum intact.

\section{Lu-1290. Ailsa, Sample 1}

$13,970 \pm 200$

$\delta^{13} \mathrm{C}=-23.2 \%$

Clay gyttja from lowermost part of sediment core from lake near Ailsa, S Hochstetter Forland $\left(75^{\circ} 19^{\prime} \mathrm{N}, 19^{\circ} 40^{\prime} \mathrm{W}\right)$. Above Late Weichselian marine limit. Comment: pretreated with HCl. Undersized; diluted; $53 \%$ sample. (3 1-day counts.)

\section{Lu-1291. Agnetesöelven, Lake A, Sample 1}

$$
\begin{array}{r}
\mathbf{8 9 0 0} \pm \mathbf{1 1 0} \\
\delta^{13} C=-24.5 \%
\end{array}
$$

Clay gyttja from lowermost part of sediment core from lake $S$ of Agnetesöelven, N Hochstetter Forland (75 $\left.35^{\prime} \mathrm{N}, 19^{\circ} 50^{\prime} \mathrm{W}\right)$. Dates deglaciation of area. Comment: pretreated with HCl. Undersized; diluted; $71 \%$ sample.

\section{Lu-1292. Ardencaple Fjord 1}

$$
\begin{array}{r}
\mathbf{7 4 5 0} \pm \mathbf{7 5} \\
\delta^{1.3} C=+0.4 \%
\end{array}
$$

Shell fragments (Mya truncata, Hiatella arctica) from base of $20 \mathrm{~m}$ thick silt deposit $6 \mathrm{~km} \mathrm{NW}$ of Kap Buch, Ardencaple Fjord $\left(75^{\circ} 12^{\prime} \mathrm{N}\right.$, $\left.20^{\circ} 35^{\prime} \mathrm{W}\right)$. Coll at $3 \mathrm{~m}$. In same level were also some shells of Portlandia arctica. Comment: outer $26 \%$ removed by acid leaching.

\section{Lu-1298. Kap Tramnitz}

$$
\begin{aligned}
& 19,000 \pm 190 \\
& \delta^{13} C=+0.8 \%
\end{aligned}
$$

Shell fragments (Hiatella arctica) from silt deposit underlying beachridge at $55 \mathrm{~m}$ ca $3 \mathrm{~km}$ NE of Kap Tramnitz, S Shannon 0 o $\left(75^{\circ} 02^{\prime} \mathrm{N}\right.$, $18^{\circ} 52^{\prime} \mathrm{W}$ ). Comment: outer $56 \%$ removed by acid leaching. (3 1-day counts.)

\section{Lu-1299. Peters Bugt}

$$
\begin{aligned}
& >42,400 \\
& \delta^{1 .} \mathrm{C}=+0.7 \%
\end{aligned}
$$

Shell fragments (Mya truncata) from laminated silt and sand underlying terminal moraine parallel to but younger than that one clated by Lu-1288, above. Coll at ca 45m, Peters Bugt, S Hochstetter Forland $\left(75^{\circ} 18^{\prime} \mathrm{N}, 20^{\circ} 00^{\prime} \mathrm{W}\right)$. Comment: outer $71_{\%}^{\prime} \%$ removed by acid leaching. (3 1-day counts.) 


\section{Lu-1300:1. Hochstetter east 1, inner fraction}

$$
\begin{array}{r}
9470 \pm 90 \\
\delta^{1.3} C=+0.3 \%
\end{array}
$$

Thick shells (Hiatella arctica) from silt at $13 \mathrm{~m}$, SE Hochstetter Forland $\left(75^{\circ} 17^{\prime} \mathrm{N}, 19^{\circ} 28^{\prime} \mathrm{W}\right)$. Comment: inner fraction (29\% of shells) was used.

\section{Lu-1300:2. Hochstetter east 1, outer fraction $\quad 9520 \pm 90$ \\ $\delta^{1.3} \mathrm{C}=+0.7 \%$}

Outer fraction of shells used for Lu-1300:1. Comment: outer fraction was $32 \%$ of shells; outermost $39 \%$ removed by acid leaching.

\section{Lu-1301. Peters Bugt Lake, Sample 1}

$12,960 \pm 235$

$\delta^{13} \mathrm{C}=-22.3 \%$

Clay gyttja from lowermost part of secliment core from lake at Peters Bugt, S Hochstetter Forland $\left(75^{\circ} 19^{\prime} \mathrm{N}, 20^{\circ} 03^{\prime} \mathrm{W}\right)$. Comment: pretreated with HCl. Small sample; diluted; $40 \%$ sample. (3 1-day counts.)

\section{Lu-1302. Hill 150}

$$
\begin{aligned}
& 33,500+1550 \\
& \delta^{13} C=+1.2^{\%} \%
\end{aligned}
$$

Shells (Hiatella arctica) from silt on top of $150 \mathrm{~m}$ high hill. Highest

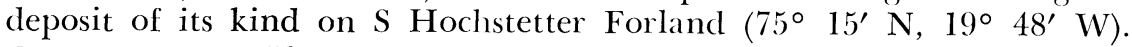
Comment: outer $53 \%$ removed by acid leaching.

\section{Lu-1303. Kildedalen}

$$
\begin{array}{r}
\mathbf{8 9 3 0} \pm \mathbf{9 0} \\
\delta^{1.3} \mathrm{C}= \pm 0.0 \% \text { }
\end{array}
$$

Thin shell fragments (Mya truncata) from deltaic sand overlying silt. Coll at 20 to $25 \mathrm{~m}$ but probably dates or closely postdates local marine limit at $50 \mathrm{~m}$ and deglaciation of outermost Kildedalen, Ardencaple Fjord $\left(75^{\circ} 15^{\prime} \mathrm{N}, 20^{\circ} 55^{\prime} \mathrm{W}\right)$. Comment: outer $53 \%$ removed by acid leaching.

\section{Lu-1304. Agnetesöelven, Lake B, Sample 1}

$7630 \pm 120$

$\delta^{1.3} \mathrm{C}=-27.2 \%$

Clay gyttja from lowermost part of sediment core from lake $\mathrm{S}$ of Agnetesöelven, N Hochstetter Forland (75 $\left.34^{\prime} \mathrm{N}, 19^{\circ} 53^{\prime} \mathrm{W}\right)$. Comment: pretreated with HCl. Small sample; diluted; 45\% sample. (3 1-day counts.)

\section{Lu-1384. Nanok}

$$
\begin{array}{r}
9810 \pm 95 \\
\delta^{13} C=+1.0 \%
\end{array}
$$

Large thick shells (Mya truncata) from silt at $30 \mathrm{~m} 3 \mathrm{~km} \mathrm{~W}$ of Nanok, $\mathrm{S}$ Hochstetter Forland $\left(75^{\circ} 10^{\prime} \mathrm{N}, 19^{\circ} 51^{\prime} \mathrm{W}\right)$. Postdates part of same terminal moraine as predates by Lu-1299, above. Comment: outer $57 \%$ removed by acid leaching. 


\section{Lu-1385. Northern Shannon}

$$
\begin{array}{r}
40,200 \\
+\mathbf{1 8 0 0} \\
\delta^{1.3} C=+1.1 \% 0 \\
=+1 \% 0
\end{array}
$$

Shells (Hiatella arctica) coll at $39 \mathrm{~m}$ on silt-covered hill reaching $48 \mathrm{~m}$, ca $4.5 \mathrm{~km} \mathrm{~N}$ of pt $54 \mathrm{~m}$ on $\mathrm{N}$ Shannon $\ddot{O}\left(75^{\circ} 18^{\prime} \mathrm{N}, 18^{\circ} 32^{\prime} \mathrm{W}\right)$. Comment: outer 52\% removed by acid leaching. (4 1-day counts.)

\section{Lu-1386. Hochstetter east 2}

$$
9400 \pm 90
$$

$$
\delta^{1.3} C=+0.8 \%
$$

Shells (Mya truncata) from sand overlying silt at $30 \mathrm{~m}$, SE Hochstetter Forland $\left(75^{\circ} 19^{\prime} \mathrm{N}, 19^{\circ} 25^{\prime} \mathrm{W}\right)$. Comment: outer $57 \%$ removed by acid leaching.

\section{Lu-1387. Hochstetter east 3}

$$
\begin{array}{r}
\mathbf{3 5 , 4 0 0}+\mathbf{1 2 0 0} \\
\delta^{13} C=+0.3 \% \\
+3050
\end{array}
$$

Shell fragments (Mya truncata, Hiatella arctica) from silt at 60 to $65 \mathrm{~m}$, SE Hochstetter Forland $\left(75^{\circ} 27^{\prime} \mathrm{N}, 19^{\circ} 23^{\prime} \mathrm{W}\right)$. Comment: outer $40 \%$ removed by acid leaching. (4 1-day counts.)

\section{Lu-1388. Hochstetter east 4}

Shells (Hiatella arctica) from sand overlain by till bed, SE Hochstetter Forland $\left(75^{\circ} 17^{\prime} \mathrm{N}, 19^{\circ} 27^{\prime} \mathrm{W}\right)$. Comment: outer $48 \%$ removed by acid leaching. (4 1-day counts.)

\section{Lu-1389. Kap Copeland}

$$
\begin{array}{r}
9370 \pm 90 \\
\delta^{1.3} C=+0.8 \% \text {. }
\end{array}
$$

Shells (Hiatella arctica) from silt reaching $41 \mathrm{~m}$ at Kap Copeland, $\mathrm{N}$ Shannon ơ $\left(75^{\circ} 20^{\prime} \mathrm{N}, 18^{\circ} 55^{\prime} \mathrm{W}\right)$. Postdating ice moving $\mathrm{S}$ along Shannon Sund. Comment: outer $64 \%$ removed by acid leaching.

\section{Lu-1390. Ardencaple Fjord 2}

$$
\begin{array}{r}
\mathbf{8 5 7 0} \pm \mathbf{8 5} \\
\delta^{13} \mathrm{C}=+0.7 \%
\end{array}
$$

Large shells (Mya truncata) from sandy silt at 30 to $40 \mathrm{~m}, 11 \mathrm{~km} \mathrm{NW}$ of Kap Buch, Ardencaple Fjord (75 $\left.13^{\prime} \mathrm{N}, 20^{\circ} 40^{\prime} \mathrm{W}\right)$. Local marine limit $50 \mathrm{~m}$. Comment: outer $60 \%$ removed by acid leaching.

\section{Merijän järvi series}

\section{Finland}

Eriophorum-Sphagnum peat from bog situated beside Lake Merijänjärvi, Ii parish, Oulu province, Finland (65 $\left.15^{\circ} 30^{\prime \prime} \mathrm{N}, 25^{\circ} 30^{\prime} 30^{\prime \prime} \mathrm{E}\right)$. Alt $30 \mathrm{~m}$. Coll 1975 and subm by M Hjelmroos, Dept Quaternary Geol, Univ Lund. Dating is part of study on vegetational development and human influence in area. Depths given are below bog surface. All samples pretreated with HCl.

Lu-1311. Merijänjärvi, 183 to $193 \mathrm{~cm}$

$$
\begin{array}{r}
\mathbf{2 7 9 0} \pm \mathbf{5 5} \\
\delta^{13} C=-26.9 \%
\end{array}
$$

First signs of human influence recorded in pollen diagram. 
Lu-1312. Merijänjärvi, 169 to $175 \mathrm{~cm}$

First Picea maximum; one grain of Plantago lanceolata.

Lu-1313. Merijänjärvi, 138 to $142 \mathrm{~cm}$

Decrease of Picea.

Lu-1314. Merijänjärvi, 110 to $115 \mathrm{~cm}$

Maximum of Urtica.

Lu-1315. Merijänjärvi, 72 to $78 \mathrm{~cm}$

Maximum of heath components.

Lu-1316. Merijänjärvi, 66 to $70 \mathrm{~cm}$

$\mathbf{2 3 2 0} \pm \mathbf{5 5}$

Further decrease of Picea.

Lu-1317. Merijänjärvi, 25 to $32 \mathrm{~cm}$

$\mathbf{1 4 3 0} \pm \mathbf{5 0}$

Rational limit of Cerealia.

\section{Pilpajärvi series}

Sediment from Lake Pilpajärvi, Oulu province, Finland (64 $57^{\prime}$ N, $25^{\circ} 49^{\prime}$ E). Alt 40m. Coll 1976 by $\mathrm{M}$ Hjelmroos and C Renaud; subm by $M$ Hjelmroos. Part of same project as Merijänjärvi series, above. Depths given are below secliment surface. Water depth at sampling point ca $5 \mathrm{~m}$. All samples pretreated with $\mathrm{HCl}$.

\section{Lu-1369. Pilpajärvi, 194 to $200 \mathrm{~cm}$}

Clay gyttja. First pollen grains of Cerealia.

Lu-1370. Pilpajärvi, 179 to $187 \mathrm{~cm}$

Gyttja. Maximum of herb components.

Lu-1371. Pilpajärvi, 156 to $164 \mathrm{~cm}$

Gyttja. Decrease of Urtica and Cercalia.

Lu-1372. Pilpajärvi, 130 to $138 \mathrm{~cm}$

Gyttja. Empiric limit of Cerealia.

Lu-1318. Kaakonlantto

$$
\begin{array}{r}
4000 \pm 60 \\
\delta^{1.3} C=-27.2 \%
\end{array}
$$

$$
\begin{array}{r}
\mathbf{3 6 6 0} \pm \mathbf{6 0} \\
\delta^{1.3} C=-28.3 \% \\
\mathbf{3 3 7 0} \pm \mathbf{5 5} \\
\delta^{1.3} C=-29 .()^{\prime} / \mathrm{C}
\end{array}
$$

$$
\begin{array}{r}
\mathbf{2 4 2 0} \pm \mathbf{5 0} \\
\delta^{13}(:-29.1 \%
\end{array}
$$

$$
\delta^{1 .}\left(\begin{array}{r}
\mathbf{9 2 0} \pm \mathbf{5 0} \\
-26 .()^{\prime} / \mathrm{co}
\end{array}\right.
$$

Peat from 220m below surface in small bog near archaeol excavatdions of Ylitornio, Tomio Lappland, Finland $\left(66^{\circ} 13^{\prime} \mathrm{N}, 23^{\circ} 16^{\prime} \mathrm{E}\right)$. 
Alt $50 \mathrm{~m}$. Coll 1975 and subm by M Hjelmroos. Dating is part of study on human settlement history by means of pollen analysis. Sample corresponds to level with earliest occurrence of pollen grains of Cerealia.

\section{Lu-1248. Oravaisensaari}

$$
\begin{array}{r}
390 \pm 70 \\
\delta^{1 .} C=-27.1 \%
\end{array}
$$

Small wood fragments from $45 \mathrm{~cm}$ below surface on Oravaisensaari I., $8 \mathrm{~km} \mathrm{~N}$ of Tornio, Finland $\left(65^{\circ} 55^{\prime} \mathrm{N}, 24^{\circ} 10^{\prime} \mathrm{E}\right)$. Alt $5.9 \mathrm{~m}$ above Tornio R. Coll 1974 and subm by M Hjelmroos. Sample comes from cultural layer, archaeol dated to Early Middle ages. Dated as part of study on human settlement history in area assoc with pollen analysis.

\section{E. Poland}

\section{Lower Vistula valley series (II)}

Marine mollusk shells from same stratigraphic level as shells previously dated in this series ( $R$, 1976, v 18, p 303-304). Coll 1976 and subm by E Drozdowski, Inst Geog, Polish Acad Sci, Toruń, Poland. Dated to solve chronologic problem assoc with redeposited mollusk fauna in lower Vistula $\mathrm{R}$ valley (Galon, 1934).

\section{Lu-1326. Mala Slońca 1, Nassa and Cardium $\quad 37,400+2000$}

$$
\delta^{1.3} C=-0.6 \%
$$

Shells (Nassa reticulata, Cardium edule) id by C Hjort, from intermorainic sandy-gravelly sediments between 2nd and 3rd morainic strata, 11 to $18 \mathrm{~m}$ above floodplain, at Mała Słońca $\left(54^{\circ} 03^{\prime} \mathrm{N}, 18^{\circ} 48^{\prime} \mathrm{E}\right)$. Comment: outer $43^{\circ}$; of shells removed by acid leaching. (4 1-day counts.)

\section{Lu-1327. Mala Slońca 1, thick fragments}

$$
\begin{aligned}
& >\mathbf{4 2 , 3 0 0} \\
\delta^{1.3} C & =-1.5 \%
\end{aligned}
$$

Thick unidentifiable bivalve fragments from same sample as Lu1326. Comment: outer $44 \%$ removed by acid leaching. (3 1-day counts.)

\section{Lu-1328. Mala Slońca 2, Nassa reticulata}

$$
\begin{array}{r}
40,700 \begin{array}{r}
+\mathbf{2 6 5 0} \\
\mathbf{2 0 0 0}
\end{array} \\
\delta^{1 .} C=-1.0 \%
\end{array}
$$

Shells (Nassa reliculata) id by C: Hjort, from layer of fine gravel, 6 to $7 \mathrm{~cm}$ thick, $5 \mathrm{~m}$ below morainic stratum and ca $20 \mathrm{~m}$ above floodplain at Mała Stoncal. Comment: outer $290 \%$ removed by acid leaching. (3 1 (lay counts.)

\section{Lu-1329. Biola Góra}

$$
\begin{aligned}
& >41,900 \\
\delta^{1.3}(i & = \pm(1) .)^{\prime} / \mathrm{C}
\end{aligned}
$$

Shells and shell fragments (Cardium edule, Ci echinatum, Macoma ballhica, Cyprina islandica, Nassa reticulata, and Bittium reticulatum) id by (: Hjort from sandy-gravelly sediments between 2nd and srel moranic strata at Biota Góa $\left(53^{\circ} 57^{\prime} \mathrm{N}, 18^{\circ} 55^{\prime} \mathrm{E}\right)$. Shells were coll ca 2 to sm above flood terrace. (iomment: outer $28 \%$, removed by acid leaching. (3 1-day counts.) 


\section{Mammoth bone series}

For other mammoth dates, see R, 1976, v 18, p 291-293 and Berglund et al (1976).

\section{Lu-1346. Bzianka}

$$
\begin{array}{r}
14,080 \pm 165 \\
\delta^{1.3} C=-20.0 \%
\end{array}
$$

Collagen from bone fragment from skull of Mammuthus primigenius from small stream near Brianka, Rzeszów, S Poland (ca $50^{\circ} \mathrm{N}, 22^{\circ} \mathrm{E}$ ). Coll 1851 by Lozinski; subm by H Kubiak, Inst Systematic and Experimental Zool, Polish Acad Sci, Cracow, Poland. For details about mammoth find, see Hauer (1851), Kulczycki (1955, PI I), and Kubiak (1965, p 17-18). Comment: collagen extracted as described previously (R, 1976, $v$ 18, p 290); no NaOH treatment. Organic carbon content: 3.3\%. Sample undersized; diluted; $71 \%$ sample. (3 1-day counts.)

\section{Lu-1347. Debica}

$$
25,300 \pm 500
$$

$$
\delta^{1.3} \mathrm{C}=-20.1 \%
$$

Collagen from bone fragment of Mammuthus primigenius, id by $\mathrm{H}$ Kubiak, from river gravels of Wisłoka $\mathrm{R}$ near Dẹbica, S Poland $\left(50^{\circ}\right.$ $10^{\prime} \mathrm{N}, 21^{\circ} 30^{\prime} \mathrm{E}$ ). Coll 1975 by schoolboys; subm by H Kubiak. Preliminary report about find pub by submitter (Kubiak, 1976). Comment: collagen extracted in same way as Lu-1346 but including $\mathrm{NaOH}$ treatment. Organic carbon content: $5.4 \%$.

$$
\text { II. ARCHAFOLOGIC SAMPLES }
$$

\section{Ingelstorp series}

Sweden

Charcoal from settlement areas and grave fields at Ingelstorp, Scania. Coll March 1974 to Nov 1976 and subm by M Strömberg, Hist Mus, Univ Lund. Preliminary report pub by submitter (Strömberg, 1977). For other dates from Ingelstorp, see R, 1976, v 18, p 314; 1977, v 19, p 435-436. All samples pretreated with $\mathrm{HCl}$ and $\mathrm{NaOH}$.

Lu-1250. Ingelstorp 10, Sample $11: 75-76$

$$
\begin{array}{r}
\mathbf{3 7 6 0} \pm \mathbf{6 0} \\
\delta^{13} \mathrm{C}=-24.8 \% \text { co }
\end{array}
$$

Charcoal from coffin in Grave 2 (1974), Ingelstorp $10\left(55^{\circ} 25^{\prime} \mathrm{N}\right.$, $14^{\circ} 03^{\prime}$ E). Assoc with flint objects. Comment (MS): date as expected.

\section{Lu-1293. Ingelstorp 13, Sample 13:75-76}

$$
\begin{array}{r}
\mathbf{2 8 3 0} \pm \mathbf{5 5} \\
\delta^{13} \mathrm{C}=-23.4 \%
\end{array}
$$

Charcoal from Hearth No. 24, Ingelstorp $13\left(55^{\circ} 25^{\circ} \mathrm{N}, 14^{\circ} 02^{\prime} \mathrm{E}\right)$. Assoc with pottery. Comment (MS): on new map property designation changed to Ingelstorp 110:1.

\section{Lu-1294. Ingelstorp 13, Sample 14:75-76}

Charcoal from Hearth No. 6, Ingelstorp 13.

$$
\begin{array}{r}
\mathbf{2 8 9 0} \pm \mathbf{5 5} \\
\delta^{13} C=-23.9 \%
\end{array}
$$


Lu-1295. Ingelstorp 13, Sample 15:75-76

$$
\begin{array}{r}
\mathbf{2 9 2 0} \pm \mathbf{5 5} \\
\delta^{13} \mathrm{C}=-23.3 \%
\end{array}
$$

Charcoal from Hearth No. 18, Ingelstorp 13. Comment (MS): 3 dates from Ingelstorp 13 agree well mutually and are of expected age judging from archaeol evidence.

\section{Lu-1321. Ingelstorp 32, Prov 1:HT76}

$$
\begin{array}{r}
3100 \pm 60 \\
\delta^{13} C=-24.7 \%
\end{array}
$$

Charcoal from pit near Grave 40 (urn grave), Ingelstorp $32^{5}\left(55^{\circ}\right.$ $\left.25^{\prime} \mathrm{N}, 14^{\circ} 03^{\prime} \mathrm{E}\right)$. Report from study of other Bronze age graves in area pub by submitter (Strömberg, 1975, p 67-69). Comment (MS): ca 700 yr older than expected.

\section{Lu-1322. Ingelstorp 32, Sample 2:HT76}

$$
\begin{array}{r}
\mathbf{4 5 8 0} \pm \mathbf{6 0} \\
\delta^{1.3} \mathrm{C}=-26.8 \%
\end{array}
$$

Charcoal from fire pit (Grave 41), Ingelstorp $32^{5}$. Comment (MS): expected to be of same age as Lu-1321.

Lu-1323. Ingelstorp 32, Sample 3 :HT76

$$
\begin{array}{r}
\mathbf{3} 1 \mathbf{5 0} \pm \mathbf{6 5} \\
\delta^{13} C=-2.7 .7 \%
\end{array}
$$

Charcoal from Grave 43 (oak trunk grave), Ingelstorp $32^{5}$. Assoc with helically ornamented bronze object. Comments: sample undersized; diluted; $82 \%$ sample (MS): somewhat older than expected.

\section{Lu-1324. Ingelstorp 325 , Sample 4:HT76}

$$
\begin{array}{r}
\mathbf{1 9 8 0} \pm \mathbf{5 0} \\
\delta^{13} C=-24.0 \%
\end{array}
$$

Charcoal from fire pit (Grave 49), Ingelstorp 325. Assoc with pottery. Comment (MS): somewhat younger than expected.

$$
\begin{aligned}
& \text { Lu-1325. Ingelstorp 32 } \text {, Sample 5:HT76 } 2720 \pm 55 \\
& \delta^{13} C=-24.0 \%
\end{aligned}
$$

Charcoal from fire pit (Grave 54), Ingelstorp 325. Assoc with pottery. Comment (MS): ca 400 yr older than expected.

\section{Lu-1348. Ingelstorp 32, Sample 6:HT76}

$$
\begin{array}{r}
\mathbf{3 1 8 0} \pm \mathbf{6 0} \\
\delta^{13} C=-23.6 \% \%
\end{array}
$$

Charcoal from stratum above Grave 63. Assoc with earthenware pots. Comments: only mild pretreatment with $\mathrm{NaOH}$ and HCl. Sample undersized; diluted; 92\% sample. (MS): date is reasonable for this Late Neolithic grave, presence of which may explain unexpected old dates for some samples from Ingelstorp $32^{5}$. Samples were originally all expected to be from Late Bronze age or Early Iron age.

Lu-1249. Ingelstorp 32 $2^{8}$, Sample 10:75-76

$$
\begin{array}{r}
\mathbf{3 0 1 0} \pm \mathbf{5 5} \\
\delta^{13} C=-26.0 \%
\end{array}
$$

Charcoal from Grave 32 (cremation grave), Ingelstorp $32^{8}\left(55^{\circ} 25^{\prime}\right.$ $\left.\mathrm{N}, 14^{\circ} 03^{\prime} \mathrm{E}\right)$. Assoc with pottery. Comment (MS): date much older than expected. 


\section{Lu-1251. Ingelstorp 32 ${ }^{8}$, Sample 12:75-76}

$$
\begin{array}{r}
1910 \pm \mathbf{5 0} \\
\delta^{13} C=-27.6 \%
\end{array}
$$

Charcoal $(25 \%)$ and soot $(75 \%)$ from fire pit (Grave $34: 1975)$, Ingelstorp $32^{8}$. Assoc with earthenware pots and spindle whorl. Comment (MS): date agrees with assoc finds.

Lu-1349. Hagestad $98^{1}$ A, Sample $7: 1976$

$$
\begin{array}{r}
4700 \pm 65 \\
\delta^{13} C=-27.0 \% 0
\end{array}
$$

Charred food remains from inside of earthenware pot (No. 18) from Early Neolithic TRB culture settlement at Hagestad $98^{1} \mathrm{~A}$, Löderup parish, Scania $\left(55^{\circ} 24^{\prime} \mathrm{N}, 14^{\circ} 11^{\prime} \mathrm{E}\right)$. Coll 1968 and subm by M Strömberg. Assoc with flint objects and other pottery. No pretreatment due to small sample size. Comment (MS): date is reasonable.

\section{Lu-1365. Simris $2^{3}$}

$$
\begin{array}{r}
\mathbf{1 9 6 0} \pm \mathbf{5 0} \\
\delta^{13} \mathrm{C}=-27.2 \% 0
\end{array}
$$

Resin from Grave 1972:2 (inhumation burial), Simris $2^{3}$, Simris parish, SE Scania $\left(55^{\circ} 32^{\prime} \mathrm{N}, 14^{\circ} 19^{\prime} \mathrm{E}\right)$. Coll 1972 and subm by B Stjernquist, Hist Mus, Univ Lund. For details about settlement sites and grave fields in Simris area, see Stjernquist $(1955,1961,1965)$. Other samples from Simris were dated by Uppsala lab (R, 1959, v 1, p 98; 1960 , v 2, p 125). Pretreated with HCl. Comment (BS): somewhat older than expected.

\section{Brunn series}

Seal bones, id by $\mathrm{E}$ Iregren, from Pitted Ware site Brunn, Ösmo parish, Södermanland (58 $\left.55^{\circ} 50^{\prime \prime} \mathrm{N}, 17^{\circ} 47^{\prime} 50^{\prime \prime} \mathrm{E}\right)$. Coll 1928 by I Schnell; subm by $\mathrm{S}$ Welinder, Univ Oldsaksamling, Oslo. All samples probably from Test Sq H18 (Schnell, 1930). Collagen extracted as described previously ( $R, 1976$, v 18, p 290), including $\mathrm{NaOH}$ treatment.

\section{Lu-1282. Brunn 1}

$$
4640 \pm 65
$$

Collagen from ribs of seal. Comment: organic carbon content: $4.0 \%$

\section{Lu-1283. Brunn 2}

$$
\begin{array}{r}
4610 \pm 65 \\
\delta^{13} C=-15.8 \%
\end{array}
$$

Collagen from ribs of seal. Comment: organic carbon content: $4.1 \%$.

\section{Lu-1284. Brunn 3}

$$
\begin{array}{r}
4650 \pm 65 \\
\delta^{1.3} C=-15.5 \%
\end{array}
$$

Collagen from ribs of seal. Comment: organic carbon content: $3.5 \%$.

\section{Korsnäs series}

Bones from Sq No. 106579 on Pitted Ware site Korsnäs, Grödinge parish, Södlermanland $\left(59^{\circ} 09^{\prime} 20^{\prime \prime} \mathrm{N}, 17^{\circ} 48^{\prime} 30^{\prime \prime} \mathrm{E}\right)$. Coll 1970 by E Baudou; subm by $\mathrm{S}$ Welinder. Collagen extracted in same way as for Brumn series, above. Bone id by K Sörensen, Zool Mus, Copenhagen. 


\section{Lu-1285. Korsnäs 1}

$$
\begin{array}{r}
\mathbf{4 1 9 0} \pm \mathbf{6 0} \\
\delta^{13} \mathrm{C}=-20.8 \%
\end{array}
$$

Collagen from bone of terrestrial animals from Layer $\mathrm{I}, 0$ to $10 \mathrm{~cm}$ below surface. Comment: organic carbon content: $3.4 \%$.

\section{Lu-1286. Korsnäs 2}

$$
\begin{array}{r}
4.270 \pm 60 \\
\delta^{1.3} C=-19.5 \%
\end{array}
$$

Collagen from bone of terrestrial animals and seal (Phoca groenlandica) from Layer II, 10 to $20 \mathrm{~cm}$ below surface. Comment: organic carbon content: $3.4 \%$.

\section{Lu-1287. Korsnäs 3}

$$
\begin{array}{r}
4560 \pm 60 \\
\delta^{13} C=-17.9 \%
\end{array}
$$

Collagen from bone, mainly of seal, from Layer III, 20 to $30 \mathrm{~cm}$ below surface. Comment: organic carbon content: $3.7 \%$.

General Comment for Brunn and Korsnäs series: dates on collagen from marine animals must be corrected because of apparent ${ }^{14} \mathrm{C}$ age of such animals during their lifetime. Size of correction is not yet known for seals in this area, but -200 to $-400 \mathrm{yr}$ may be a fair estimate of expected range.

\section{Hjulberga series}

Charcoal and carbonized hazel-nut shells from Early Neolithic TRB site Hjulberga 1, Eker parish, Närke (59॰2 $\left.21^{\prime} \mathrm{N}, 15^{\circ} 07^{\prime} \mathrm{E}\right)$. Coll 1976-77 and subm by $S$ Welinder.

\section{Lu-1319. Hjulberga $1:$ A, Sample 7}

$$
\begin{array}{r}
4830 \pm 65 \\
\delta^{13} C=-22.2 \%
\end{array}
$$

Carbonized hazel-nut shells coll from excavated trench. Comment: pretreated with $\mathrm{HCl}$ and $\mathrm{NaOH}$.

\section{Lu-1320. Hjulberga 1 :A, Sample 8}

$$
\begin{array}{r}
24.60 \pm 95 \\
\delta^{13} \mathrm{C}=-24.8 \% \text { \% }
\end{array}
$$

Charcoal and bark of Pinus, id by $\mathrm{T}$ Bartholin, from Pit W18, containing also main part of Sample 7, above. Comment: no pretreatment; sample undersized; diluted; $33 \%$ sample. (3 1-day counts.)

\section{Lu-1434. Hjulberga 1 :A, Sample 9}

$$
\begin{array}{r}
\mathbf{4 3 4 0 0} \pm \mathbf{8 0} \\
\delta^{1.3} \mathrm{C}=-22.4 \%
\end{array}
$$

Carbonized hazel-nut shells sieved from large sample from basal part of culture layer. Comment: no pretreatment; sample undersized; diluted; $48 \%$ sample. (3 1-dlay counts.)

General Comment (SW): Lu-1320 much too late like 3 other dates on charcoal from same site dated at Stockholm lab: St 5396, 1985 \pm 100 ; St 5397, 3370 \pm 100 ; St 5398, $590 \pm 105$. Excavations unpub (cf Bagge, 1949). 
Lu-1350. Nordansjö 1:5

$$
\begin{array}{r}
1060 \pm 135 \\
\delta^{13} C=-24.9 \%
\end{array}
$$

Wood fragments (Betula sp) id by $\mathrm{T}$ Bartholin from hole for handle in iron-ax exposed by wave action at shore of Lake Malgomaj, Site Raä 756, Nordansjö 1:5, Vilhelmina parish, Lappland $\left(64^{\circ} 42^{\prime} \mathrm{N}, 16^{\circ} 22^{\prime} \mathrm{E}\right.$; Sw Grid Ref x7177,2; y 1527,0$)$. Shore erosion caused by storage-capacity regulation of lake. Coll 1975 by B Eriksson; subm by A Huggert, Västerbottens Mus, Umeå. Comment: no pretreatment; very small sample; diluted; $15 \%$ sample. (4 1-day counts.)

\section{Karlshacka series}

Charcoal from Site Raä 572, Karlsbacka 1:2, Vilhelmina parish, Lappland $\left(64^{\circ} 41^{\prime} \mathrm{N}, 16^{\circ} 27^{\prime} \mathrm{E}\right.$; Sw Grid Ref x7175,8; y1530,6). Coll 1976 by K Wijkander; subm by A Huggert.

\section{Lu-1351. Karlsbacka 1:2, Sample 1}

$$
\begin{array}{r}
160 \pm 45 \\
\delta^{13} C=-25.5 \% \text { \% }
\end{array}
$$

Charcoal from hearth above layer of cracked stones. Comment: no pretreatment; small sample.

\section{Lu-1352. Karlsbacka $1: 2$, Sample 2}

$$
\begin{array}{r}
220 \pm 70 \\
\delta^{13} C=-26.4 \% \text { o }
\end{array}
$$

Charcoal from upper part of rust-colored soil below layer of cracked stones. Comment: no pretreatment; undersized; diluted; $51 \%$ sample.

\section{REFFRFNCES}

Bagge, A, 1949, Snörkeramikboplatsen vid Rosenlund, Hjulberga, Ekers sn, Närke: Fornvännen (Stockholm).

Berglund, B E, 1971, Late-Glacial stratigraphy and chronology in South Sweden in the light of biostratigraphic studies on Mt. Kullen, Scania: Geol Fören Stockholm Förh, v 93, p $11-45$.

Berglund, B E, Håkansson, Sören, and Lagerlund, Erik, 1976, Radiocarbon-dated mammoth (Mammuthus primigenius Blumenbach) finds in South Sweden: Boreas (Oslo), v 5, p 177-191.

Galon, R, 1934, Dolina dolnej Wisly, jej ksztalt i rozwój na tle budowy dolnego Powiśla (in Polish with German summary): Badania Geog nad Pol Pn-Zach (Poznaí), v $11-12,112$ p.

Håkansson, Sören, 1972, University of Lund radiocarbon dates V: Radiocarbon, v 14, p $380-400$. p $493-513$.

1974, University of Lund radiocarbon dates V'II: Radiocarbon, v 16 , p $307-330$. p $174-195$. p $290-320$.

1976 , University of Lund radiocarbon dates IX: Radiocarbon, v 18 ,

1977, University of Lund radiocarbon dates X: Radiocarbon, v 19, p 424-441.

Hauer, F v, 185l, Fossiler Elephantenschädel ron Bzianka bei Rzeszów: Jb d Geol Reichsanstalt, Wien, v 2, p 158-159. 
Hjort, Christian, 1973, A sea correction for East Greenland: Geol Fören Stockholm Förh, v 95, p 132-134.

Kubiak, Henryk, 1965, Slonie kopalne Polski poludniowej (in Polish with English summary): Folia Quaternaria (Kraków), no. 19, p 1-43.

1976, Mamut z Debicy: Gazeta Poludn (Kraków), no. 54, p 5.

Kulczycki, J, 1955, Les ossements des mammouths: Paleont Pol (Warszawa), v 7, p 1-65.

Olsson, Ingricl, 1959, Uppsala natural radiocarbon measurements I: Am Jour Sci Radiocarbon Suppl, v I, p 87-102.

1960, Uppsala natural radiocarbon measurements II: Am Jour Sci Radiocarbon Suppl, v 2, p 112-128.

Schnell, I, 1930, Södertörn under stenåldern: Bidrag till Södermanlands äldre kulturhistoria. No. 24, Strängnäs.

Stjernquist, B, 1955, Simris. On cultural connections of Scania in the Roman Iron age: Acta Archacol Lundensia, ser in $4^{\circ}$, no. 2, 1955, $186 \mathrm{p}$.

1961, Simris II. Bronze age problems in the light of the Simris excavation: Acta Archaeol Lundensia, ser in $4^{\circ}$, no. 5, 1961, 173 p.

1965, An Early Neolithic settlement site at Simris in S.E. Scania: Lunds Univ Hist Mus Medd 1964-1965, p 32-70.

Strömberg, M, 1975, Bronsålder på Österlen. Undersökningar i Valleberga-LöderupIngelstorp: Kulturnämuden i Ystad $1975,80 \mathrm{p}$

1977, 84 p. 